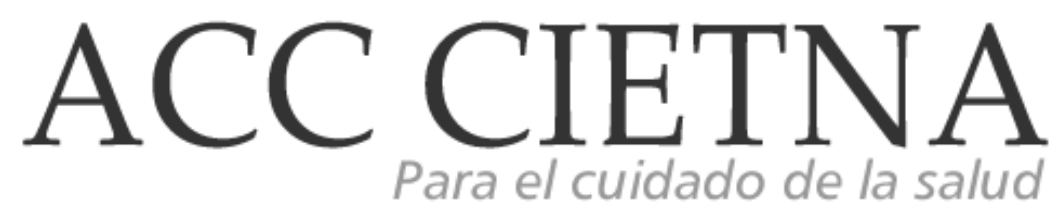

https://doi.org/ $10.35383 /$ cietna.v6i2.250

ARTÍCULOS DE INVESTIGACIÓN ORIGINALES

\title{
Calidad de vida de las personas con discapacidad física en su Centro Laboral, Chiclayo-2015
}

\author{
Mundaca Prado, Eliana Katherine ${ }^{1}$, Estela Flores, Darnell Karolayn de Guadalupe ${ }^{2}$, Chú \\ Montenegro, Magaly del Rosario 3
}

INFORMACIÓN DEL ARTÍCULO RESUMEN

\section{Historia del artículo:}

Recibido el 10 de septiembre de 2019

Aceptado el 09 de noviembre de 2019

Palabras clave:

Calidad de vida

Personas con discapacidad

Empleo
La calidad de vida es el factor prioritario en el cuidado de enfermería, donde va contribuir a la mejora de la salud mental, física y emocional de la persona con discapacidad física, que en muchas ocasiones son vulnerados por la sociedad, es por ello que se realizó el trabajo de investigación titulado: "Calidad de vida de las personas con Discapacidad Física en su centro laboral, Chiclayo - 2015, siendo su objetivo describir la calidad de vida, de personas con discapacidad física en su centro laboral. Se citó tres autores principales, Marriner T, Palomino G y Sebastián M. El tipo de investigación fue cuantitativo, de diseño descriptivo, se formuló la pregunta de investigación ¿Cómo es la calidad de vida de las personas con discapacidad física en su centro laboral, Chiclayo 2015?, se tuvo una población de 405 personas y una muestra de 100 personas a quienes se les aplicó el cuestionario GENCAT el cual evaluó la calidad de vida a través de 69 ítems, se tomó en cuenta los principios bioéticos de Elio Sgreccia y los principios de rigor científico según Pólit. El análisis de los datos se realizó mediante la estadística descriptiva donde se halló frecuencias absolutas y porcentajes cuya distribución se presentó a través de tablas y gráficos, utilizado los softwares SPSS, Excel y Megastat. Se llegó a la conclusión que las dimensiones Autodeterminación, R. Interpersonales, D. Personal e Inclusión social, se encuentran antes de la media estándar esperada; sin embargo, las dimensiones B. Emocional, B. Físico, B. Material y Derechos se encuentran por debajo de la media estándar esperada (10 DE +-3$)$, es decir son las más afectadas en su

\footnotetext{
${ }^{1}$ Licenciada en Enfermería en el Centro de Salud La Ramada, Llama, Chota, Cajamarca, Perú. Email: emundacap@outlook.com ${ }^{2}$ Licenciada en Enfermería en Clínica SABIA, Chiclayo, Perú. Email: $\underline{\text { darnellusat@outlook.es }}$

${ }^{3}$ Mgtr. en Enfermería / Docente de la Universidad Católica Santo Toribio de Mogrovejo, Chiclayo, Perú. Email: mchu@usat.edu.pe. ORCID: https://orcid.org/0000-0002-7707-1937
} 
calidad de vida, encontrado así dificultades en el desarrollo biopsicosocial de las personas con discapacidad física.

Occupational diseases of nursing staff and their relationship with working conditions. Provincial hospital Docente Belén de Lambayeque. 2018

\section{ABSTRACT}

\section{Keywords:}

Quality of life

People with disabilities

Employment
Quality of life is the priority factor in nursing care, where it will contribute to the improvement of mental, physical and emotional health of the person with physical disability, which in many cases are violated by society, which is why Carried out the research work entitled: "Quality of life of people with physical disabilities in their workplace, Chiclayo - 2015, and their objective is to describe the quality of life of people with physical disabilities in their workplace. Three main authors, Marriner T, Palomino $\mathrm{G}$ and Sebastián M. were cited, the type of research was quantitative, descriptive design, the research question was How is the quality of life of people with physical disabilities in their workplace, Chiclayo 2015?, A population of 405 people and a sample of 100 people to whom the GENCAT questionnaire was applied, which evaluated the quality of life through 69 items, took into account the bioethical principles of Elio Sgreccia and the principles Of scientific rigor according to Pólit. Data analysis was performed using descriptive statistics where absolute frequencies and percentages were found. The distribution was presented through tables and graphs, using SPSS, Excel and Megastat software. It was concluded that the dimensions Self-determination, R. Interpersonal, D. Personal and Social Inclusion, are before the expected average standard; However, the dimensions B. Emotional, B. Physical, B. Material and Rights are below the expected average standard (10DE +-3$)$, that is to say they are the most affected in their quality of life, thus encountered difficulties in The bio-psychosocial development of people with physical disabilities

\section{Introducción}

Enfermería es una profesión dedicada al servicio y cuidado holístico de los seres humanos, el cual tiene como prioridad la valoración de las necesidades de la persona para su óptimo bienestar, enfocándose en todos los contextos del desarrollo humano'. El cuidado enfermero no siempre va dirigido a la persona enferma $u$ hospitalizada, sino que también brinda su cuidado a la familia y a la comunidad, es por ello que la enfermera y el personal de salud deben de enfocarse en la integración socio-laboral de la persona, más aún cuando existe en la actualidad personas que padecen algún tipo de discapacidad. La persona con discapacidad es aquella que tiene una o más deficiencias físicas, mentales o sensoriales, que al interactuar con diversas situaciones en el entorno se encuentra con impedimentos ${ }^{2}$. La persona con discapacidad física encuentra mayor limitación es sus actividades diarias, ya que no puede desenvolverse con facilidad dentro de su entorno, debido a que no se le brindan espacios adecuados, implementos necesarios para su adecuado desenvolvimiento, exponiéndose inclusive a riesgos laborales. 
Así mismo, a las personas con discapacidad se les torna difícil poder integrarse a la sociedad con facilidad y más aún si se encuentran en una edad laboralmente activa, siendo el trabajo un elemento fundamental para el desarrollo y el desenvolvimiento de toda persona, sin embargo, hoy en día es frecuente ver la discriminación y el rechazo de la sociedad ante las personas con discapacidad que anhelan desenvolverse laboralmente viéndose vulnerados sus derechos.

Es por ello que la ley que ampara al discapacitado Ley General de la persona con Discapacidad $\mathrm{N}^{\circ}$ 29973, manifiesta que toda persona con discapacidad tiene derecho a trabajar en igualdad de condición, en un trabajo libremente elegido o aceptado, con igualdad de oportunidad, con condiciones de trabajo justas, seguras $y$ saludables ${ }^{2}$.

Por otro lado, las entidades públicas están en la obligación de contratar a personas con discapacidad en una proporción no menor del $5 \%$ y los empleadores privados con más de cincuenta trabajadores en una proporción no inferior a $3 \% 2$.

Según el Instituto Nacional de Estadística e Informática (INEI), en nuestro país el $5.2 \%$ de la población (1 millón 575 mil personas) presentan discapacidad. De este total, el $52,1 \%$ son mujeres que equivale a 820 mil 731 personas y $47,9 \%$ son hombres (754 mil 671) es decir, es mayor la proporción de mujeres que de hombres. Además, el estudio mostro que la discapacidad motora física es la que más afecta a la población, alcanzando 932 mil personas (59,2\%).

A nivel departamental presentan una mayor incidencia de discapacidad física la ciudad de Lima con un $6.8 \%$ de la población, seguido de Arequipa (6.7\%) y Moquegua (6,6\%), por otro lado, los departamentos de menor población de personas discapacitadas son Loreto (3.2\%), Amazonas (3.3\%) y Lambayeque con $3.5 \% 3$.

INEI también menciona que de cada 100 personas de 14 años y más edad con alguna discapacidad, 22 se encuentra trabajando, es decir, pertenecen a la población económicamente activa. En tanto, que el $76,8 \%$ corresponde a la población económicamente inactiva.

Según el área de residencia, en el área urbana, el $21,0 \%$ de las personas que presentan algún tipo de limitación forman parte de la población económicamente activa. Sin embargo, existe un $77.3 \%$ que está afuera de este segmento. 4

La calidad de vida comprende las dimensiones emocionales, físicas, materiales, relaciones interpersonales, desarrollo personal, autodeterminación, inclusión social y derechos 5 . La calidad de vida se constituye en un factor importante dentro del centro laboral, ya que le brinda comodidad, confianza a la persona con discapacidad física, dado que las personas con discapacidad son una población vulnerable que necesitan de la ayuda de otros, ya sea de manera directa e indirecta, donde muchas veces se ha evidenciado la no disposición de los empleadores, de proporcionarles las condiciones necesarias que le ayuden a salvaguardar su calidad de vida dentro de su medio laboral como: ambientes y dispositivos adecuados y óptimos para el desempeño de sus labores.

A nivel latinoamericano los autores Cardona D, Agudelo A, Restrepo L, Sánchez L y Segura ${ }^{6}$, en su investigación titulada "Calidad de vida de las personas en situación de discapacidad física", tuvo como resultado según la escala de calidad de vida relacionada con la salud, el $40 \%$ (70) y el $6.3 \%$ (11) indica tener buena y muy buena calidad de vida.

Gomez L, Verdugo M, Arias B y Irurtia J7, en su estudio titulado "Integración laboral y discapacidad", obtuvo como resultados que los trabajadores que participaron en el programa ECA, como grupo, obtuvieron puntuaciones elevadas en todas las escalas del cuestionario, y también, puntuaciones altas en la calidad de vida 
considerada globalmente. La puntuación media de la calidad de vida de los trabajadores es de 97,61 siendo la puntuación mínima de 55 y la máxima de 119 (escala: puntuación mínima de 40 y máxima de 120), representando la máxima como una calidad de vida adecuada y la mínima inadecuada.

Herrero $\mathrm{M}$ y Elias $\mathrm{V}^{8}$, en su investigación "Calidad de vida en Personas con Discapacidad en la Provincia de Huesca", obtuvo como resultados que las personas con discapacidad física obtuvieron unas puntuaciones nivel de autodeterminación como muy bueno (percentil 90), satisfacción deficiente (percentil 25), pertenecía social bueno (percentil 50).

De acuerdo a la problemática expuesta, se formuló la pregunta de investigación, ¿Cómo es la calidad de vida de las personas con discapacidad física en su centro laboral?, formulando así el objetivo general: "Describir la calidad de vida de las personas con discapacidad física en su centro laboral"; el objetivo específico "Describir cada una de las dimensiones de la Calidad de Vida de las personas con discapacidad física en su centro laboral".

La presente investigación se justificó porque actualmente las personas con discapacidad física no son reconocidas ante la sociedad, omitiéndole sus derechos como tal, asimismo no cuentan con bases informativas sobre la salud ocupacional orientada a la calidad de vida dentro de su centro laboral, debido a que las entidades públicas y privadas no facilitan un entorno adecuado en donde la persona pueda desarrollarse eficazmente. Es por ello que los resultados de la investigación serán de vital importancia, tanto para las instituciones públicas como privadas, para contribuir al mejoramiento de la calidad de vida de las personas con discapacidad física en su centro laboral, además servirá de ayuda para los estudiantes de la carrera de enfermería a tomar en cuenta esta problemática que aqueja a la sociedad, así mismo será un precedente para futuras investigaciones.

\section{Metodología}

La investigación fue de tipo cuantitativo, método descriptivo, y corte transversal, dado que permitirá la medición de las variables, usando magnitudes numéricas que pueden ser tratadas mediante la estadística ${ }^{9}$, permitiendo así analizar los datos.

La población estuvo conformada por 405 personas discapacitadas inscritas en el Consejo Nacional para la Integración de la persona con discapacidad (CONADIS) ${ }^{10}$, quienes cuentan criterios de inclusión: personas mayores de 18 años con discapacidad física que se encuentran laborando en las diversas instituciones públicas y privadas en la ciudad de Chiclayo, en un periodo mínimo de 2 meses.

La muestra fue de 100 personas, obtenida por la formula finita. El tipo de muestreo fue probabilístico, aleatorio simple.

Como técnica se utilizó la encuesta y como instrumento el cuestionario, basada en la escala GENCAT, la cual ha sido aplicada en países de España (Barcelona, Catalunya, Salamanca), EEUU, Japón y nueva Zelanda. Con respecto a Perú se ha aplicado a personas con cirugía cardiaca, con diabetes mellitus y ostomizadas, en la ciudad de Lima, y a nivel local ha sido aplicada en la Universidad Católica Santo Toribio de Mogrovejo ${ }^{11}$. El cuestionario consta de 69 preguntas cerradas, con cuatro opciones ("nunca o casi nunca", "a veces", "frecuentemente" y "siempre o casi siempre").

Para validar el instrumento en nuestro medio se realizó una prueba piloto a una población de 70 personas las cuales compartían las mismas características de la población en estudio, quienes no fueron considerados como sujetos de investigación. Obteniéndose, de acuerdo a la 
medida de consistencia interna "Alfa de crombach" un resultado de 0.824 , lo cual demuestra un alto grado de confiabilidad de que los resultados obtenidos permitirán medir las variables de estudio.

El procedimiento de recogida de datos consistió en inscribir el proyecto de investigación en el catálogo de tesis de la escuela de Enfermería, luego se presentó al comité metodológico de dicha escuela para las sugerencias y posterior aprobación. La recogida de datos se realizó en un lugar adecuado, sin distractores, lo cual permitió que el investigado responda libremente el cuestionario, en un tiempo promedio de $15 \mathrm{~min}$.

El procesamiento de datos se realizó con la ayuda del programa de Excel-2010, mediante un proceso de análisis de la variable, asimismo los datos obtenidos se vaciaron a tablas predeterminadas por la Escala de GENCAT y se presentó a través de tablas y gráficos estadísticos de acuerdo a los objetivos planteados en la investigación.

En el desarrollo de la investigación se tomó en cuenta los principios bioéticos ${ }^{12}$ de Sgreccia $E$ : principio de libertad, en el cual se respetará la libertad de la persona en decidir su participación en el estudio; principio de la sociabilidad y subsidiariedad: Esta investigación estará enfocada a difundir los resultados del estudio en las instituciones implicadas a las personas con discapacidad física. Así mismo, se tuvieron en cuenta los principios de rigor científico: Confiabilidad y de objetividad ${ }^{3}$.

\section{Resultados, análisis y discusión}

Los resultados de la investigación se encuentran en tablas, gráficas, y además el análisis y discusiones de los mismos contrastados con antecedentes obtenidos de la investigación, bibliografía de principales autores y artículos científicos.

\section{ÍNDICE DE CALIDAD DE VIDA DE LAS PERSONAS CON DISCAPACIDAD FÍSICA EN SU CENTRO LABORAL, CHICLAYO - 2015}

\begin{tabular}{|c|c|c|c|c|c|c|c|c|c|c|}
\hline \multicolumn{10}{|c|}{ PERFILE CALIDAD DEVIDA } \\
\hline Percentil & BE & RI & BM & DP & BF & AU & IS & DR & Indice de CV & Percentil \\
\hline 99 & $16-20$ & $16-20$ & $16-20$ & $16-20$ & $16-20$ & $16-20$ & $16-20$ & $16-20$ & $>130$ & 99 \\
\hline 95 & 15 & 15 & 15 & 15 & 15 & 15 & 15 & 15 & $122-130$ & 95 \\
\hline 90 & 14 & 14 & 14 & 14 & 14 & 14 & 14 & 14 & $118-121$ & 90 \\
\hline 85 & 13 & 13 & 13 & 13 & 13 & 13 & 13 & 13 & $114-117$ & 85 \\
\hline 80 & & & & & & & & & $112-113$ & 80 \\
\hline 75 & 12 & 12 & 12 & 12 & 12 & 12 & 12 & 12 & $110-111$ & 75 \\
\hline 70 & & & & & & & & & $108-109$ & 70 \\
\hline 65 & 11 & 11 & 11 & 11 & 11 & 11 & 11 & 11 & $106-107$ & 65 \\
\hline 60 & & & & & & & & & $104-105$ & 60 \\
\hline 55 & & & & & & & & & $102-103$ & 55 \\
\hline 50 & 10 & 10 & 10 & 10 & 10 & 110 & 10 & 10 & $100-101$ & 50 \\
\hline 45 & & & & & & & & & $98-99$ & 45 \\
\hline 40 & & & & & & & & & $96-97$ & 40 \\
\hline 35 & 9 & 9 & 9 & 9 & 9 & 9 & 9 & 9 & $94-95$ & 35 \\
\hline 30 & & & & & & & & & $92-93$ & 30 \\
\hline 25 & 8 & 8 & 8 & 8 & 8 & 8 & 8 & 8 & $89-91$ & 25 \\
\hline 20 & & & & & & & & & $86-88$ & 20 \\
\hline 15 & 7 & 7 & 7 & 7 & 7 & 7 & 7 & 7 & $84-85$ & 15 \\
\hline 10 & 6 & 6 & 6 & 6 & 6 & 6 & 6 & 6 & $79-83$ & 10 \\
\hline 5 & 5 & 5 & 5 & 5 & 5 & 5 & 5 & 5 & $68-78$ & 5 \\
\hline 1 & $1-4$ & $1-4$ & $(1-4)$ & $1-4$ & $1-4$ & $1-4$ & $1-4$ & $(1-4)$ & $<68$ & 1 \\
\hline & & & & & & & \\
\hline
\end{tabular}

Perfl de Calidad de vida de la Escala GENCAT

\section{Interpretación:}

En la presente tabla se muestra que las dimensiones: Autodeterminación, Relaciones Interpersonales e inclusión social, se encuentran dentro de la media: 10 DE $(+/-3)$ del índice de calidad de vida esperada. Mientras que las dimensiones Bienestar emocional, Bienestar material, Bienestar físico y Derechos se encuentran por debajo de la media estándar esperada de la Calidad de Vida. 


\section{DINENSIONES DE LA CALIDAD DE VIDA}

GRÁFIC0 N1. Calidad de Vida de las personas con Discapacidad Física en su Centro Laboral, según Bienestar Emocional, Chiclayo-2015

$\downarrow$

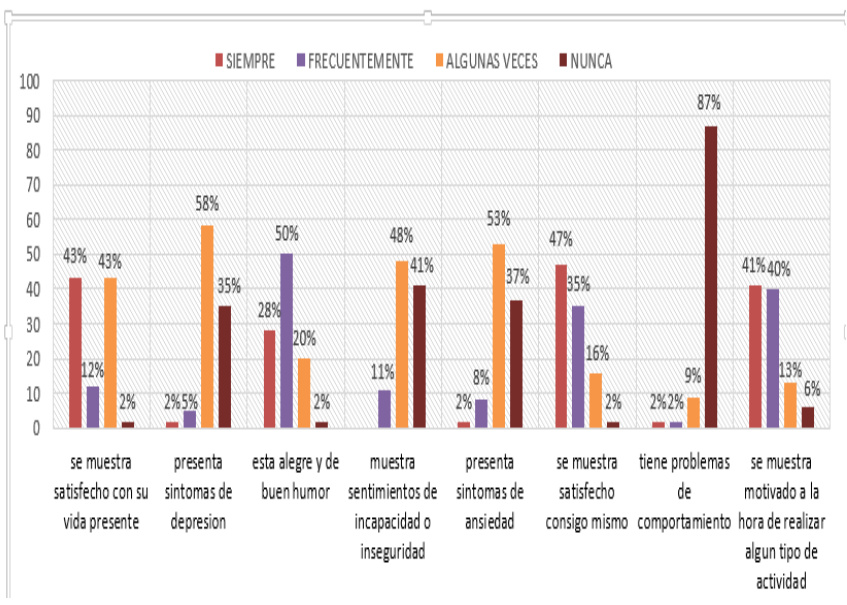

Fuente: Manual de aplicación de la Escala GENCAT de calidad de vida

\section{Interpretación:}

Se realizó el análisis de la dimensión Bienestar Emocional en donde el $45 \%$ de las personas con discapacidad física manifiestan que Algunas veces y Nunca se muestran satisfechos con su vida presente, asimismo el 59\% de discapacitados Frecuentemente y Algunas veces muestran sentimientos de incapacidad e inseguridad.

GRAFICO N2. Calidad de Vida de las personas con Discapacidad Física en su Centro Laboral, según Bienestar Físico, Chiclayo-2015 - nunCa algunasvees a frecuentemente islempre

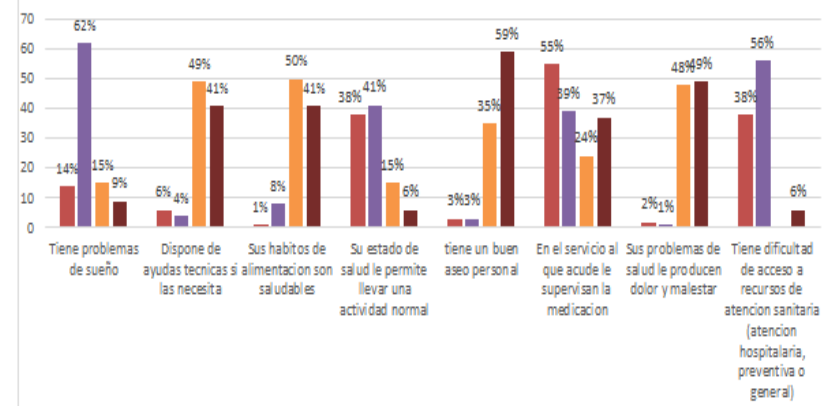

Fuente: Manual de aplicación de la Escala GENCAT de calidad de vida

\section{Interpretación:}

Se realizó el análisis de la dimensión Bienestar Físico donde el $97 \%$ de las personas con discapacidad física siempre sus problemas de salud producen dolor y malestar, además el $56 \%$ de la población algunas veces tienen dificultad de acceso a recursos de atención sanitaria.

\section{GRÁFIC0 No3. Calidad de Vida de las personas con Discapacidad Física en su Centro Laboral, según Bienestar .Vaterial, Chiclayo-2015}

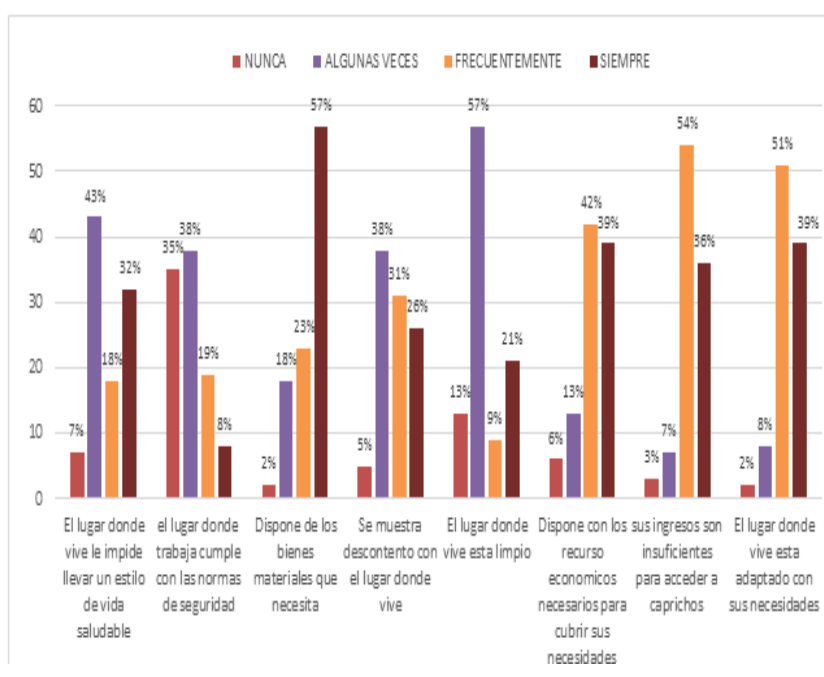

Fuente: Manual de aplicación de la Escala GENCAT de calidad de vida

\section{Interpretación:}

Se realizó el análisis de la dimensión Bienestar Material donde el $73 \%$ Nunca y Algunas veces el lugar donde trabaja cumple con las normas de seguridad, además el $70 \%$ de la población Nunca y algunas veces el lugar donde vive está limpio.

Por otro lado, el 81 \% de la población en estudio Siempre dispone con los recursos económicos necesarios para cubrir sus necesidades básicas.

\section{GRÁFIC0 No 4. Calidad de Vida de las personas con Discapacidad Física en su Centro Laboral, según Dimensión Relaciones Interpersonales, Chiclayo-2015} Fuente: Manual de aplicación de la Escala GENCAT de Calidad de Vida

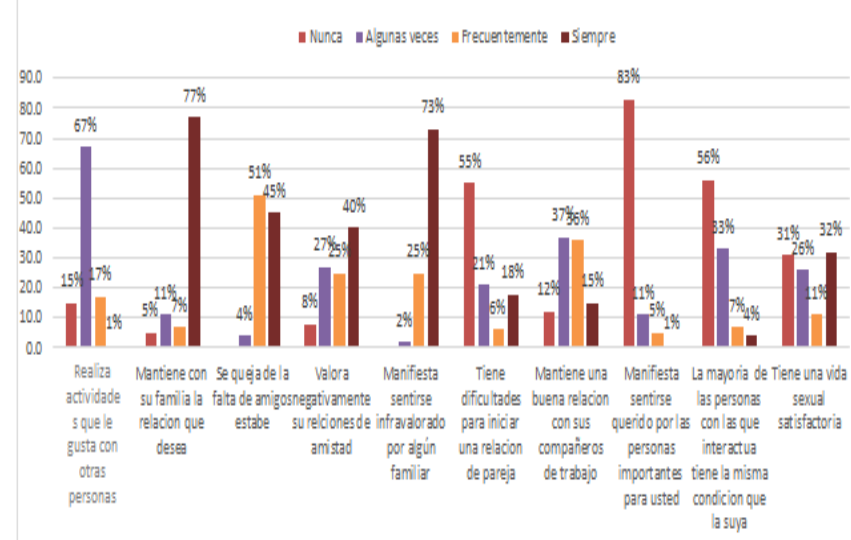

Fuente: Manual de aplicación de la Escala GENCAT de calidad de vida

\section{Interpretación:}

Se realizó el análisis de la dimensión Relaciones Interpersonales, en donde el $98 \%$ de las personas con 
discapacidad física Siempre mantiene sentirse infravalorado por algún y el $82 \%$ de la población en estudio Nunca y Algunas veces realiza actividades que le gusta con otras personas.

GRAFICO N 5.Calidad de Vida de las personas con Discapacidad Física en su

Centro Laboral, según Dimensión Desarrollo Personal, Chiclayo- 2015

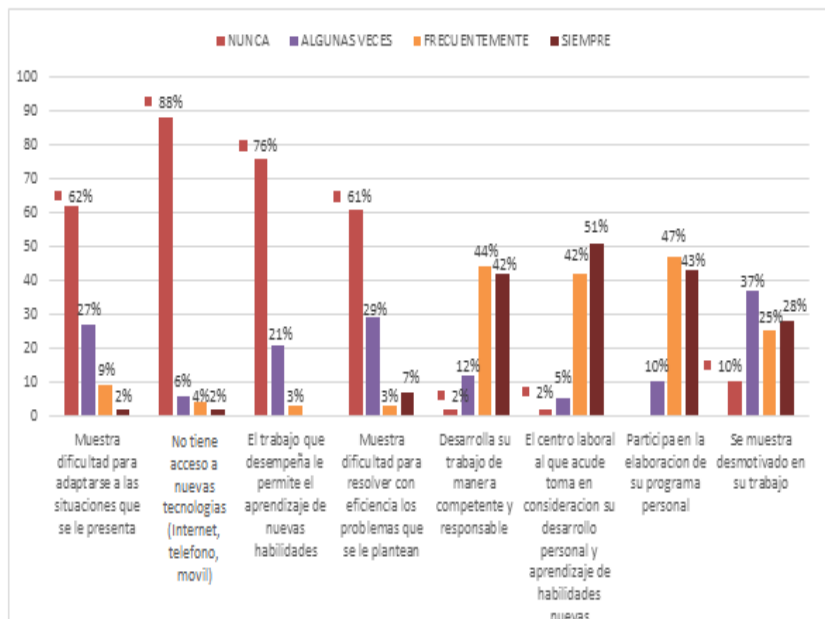

Fuente: Manual de aplicación de la Escala GENCAT de calidad de vida

\section{Interpretación:}

Se realizó el análisis de la dimensión Desarrollo Personal en donde el $87 \%$ de la población Nunca y algunas veces el trabajo que desempeña le permite el aprendizaje de habilidades nuevas y el $53 \%$ manifiesta que frecuentemente y siempre se muestran desmotivados en su trabajo.

GRÁFICO No6.Calidad de Vida de las personas con Discapacidad Física en su Centro Laboral, Dimensión Inclusión Social, Chiclayo-2015

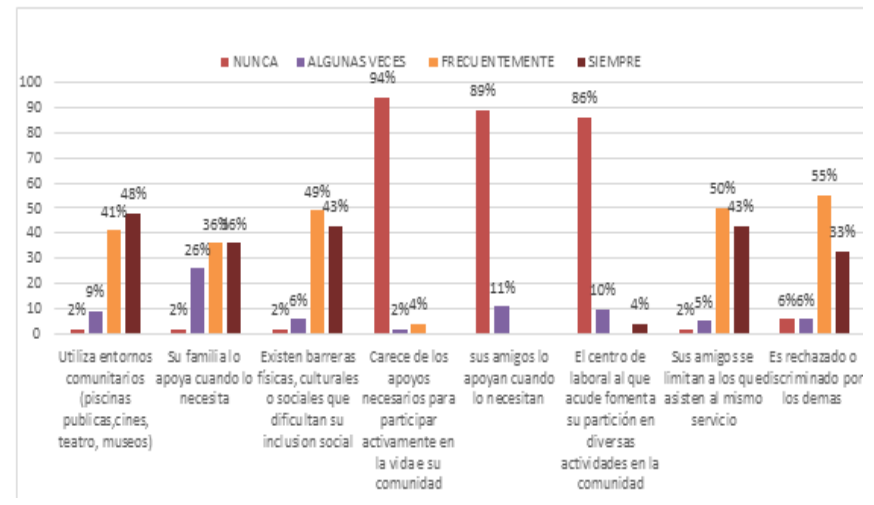

Fuente: Manual de aplicación de la Escala GENCAT de calidad de vida

\section{Interpretación:}

Se realizó el análisis de la dimensión Inclusión social en donde el $92 \%$ de las personas con discapacidad física Frecuentemente y Siempre existen barreras físicas, culturales y sociales que dificultan la inclusión social, el $89 \%$ de la población Nunca sus amigos lo apoyan cuando lo necesitan y el $88 \%$ manifiesta Siempre es rechazado o discriminado por los demás.

GRAFICO N07. Calidad de Vida de las personas con Discapacidad Física en su

Centro Laboral, según Dimensión Autodeterminación, Chiclayo-2015

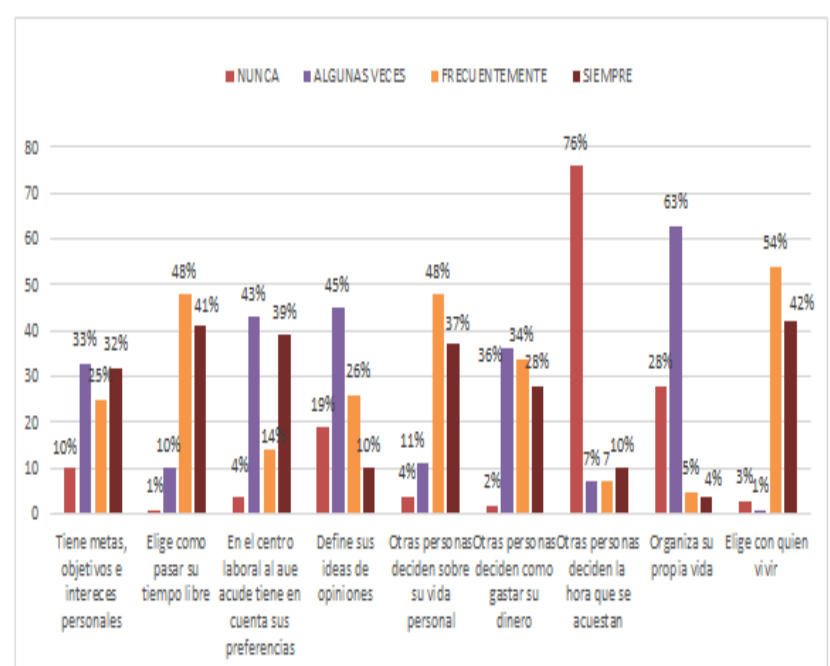

Fuente: Manual de aplicación de la Escala GENCAT de calidad de vida

\section{Interpretación:}

Se realizó el análisis de la dimensión Autodeterminación, en donde el $80 \%$ manifiesta que frecuentemente y siempre otras personas deciden sobre su vida personal, y el $91 \%$ nunca y algunas veces organiza su propia vida.

GRAFICO No8. Calidad de Vida de las personas con Discapacidad Física en su Centro Laboral, según Dimensión Derechos, Chiclayo-2015.

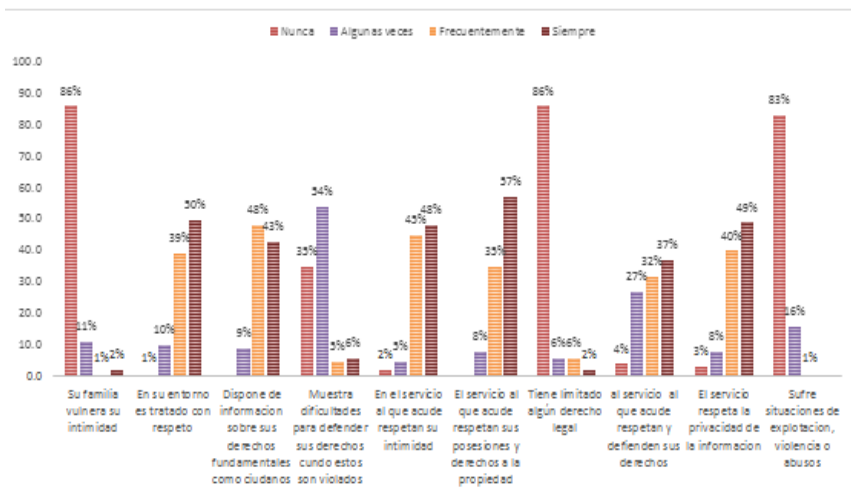

Fuente: Manual de aplicación de la Escala GENCAT de calidad de vida

\section{Interpretación:}

Se Realizó el análisis de la dimensión Derechos en donde el 48\% Frecuentemente si disponen de información sobre sus derechos fundamentales como ciudadanos y el $37 \%$ de las personas mencionan que al trabajo a donde acuden Siempre defienden y respetan sus derechos. 
Por otro lado, solo el $54 \%$ Algunas veces muestran dificultades para defender sus derechos.

\section{ANÁLISIS Y DISCUSIÓN:}

La calidad de vida es el producto de la interacción dinámica entre las condiciones externas de un individuo y la percepción interna de los mismos ${ }^{14}$; de esta manera es que surge la preocupación del sistema de salud, teniendo como prioridad elevar el estado de salud físico, emocional y psicológico de la persona, es por ello que la calidad de vida se constituye como el tema de discusión principal, pues incide directamente en el bienestar multidimensional de la persona con discapacidad fisica.

La calidad de vida influye de manera directa en el desarrollo integral y funcional de la persona con discapacidad física, conllevando a un desarrollo óptimo ante la sociedad, así como mantener un adecuado equilibrio de las dimesnsiones de vida. De esta manera, se interpretó el Gráfico $\mathrm{N}^{\circ} 1$ Dimension Bienestar Emocional donde se concluyó que el $59 \%$ de las personas con discapacidad física Frecuentemente $\mathrm{y}$ algunas veces muestran sentimientos de incapacidad $o$ inseguridad y el $45 \%$ solo Algunas veces y nunca se muestran satisfechos con su vida presente, para Latorre A. las personas con discapacidad fisica llegan a tener un sentimiento de incapacidad e inseguridad que amenaza su vida, ${ }^{15}$ puesto que todas las personas que atraviesan situaciones impactantes presentan un desequilibrio emocional, fisico y psicologico el cual conlleva a cuadros depresivos que influye en el ejercicio laboral.

Asimismo en el presente estudio se obtuvo que el 58\% Algunas veces presentan síntomas de depresión, es por ellos que la Organización Mundial de la Salud (OMS), considera a la depresión es un trastorno mental frecuente, que se caracteriza por la presencia de tristeza, pérdida de interés o placer, sentimientos de culpa o falta de autoestima, trastornos del sueño o del apetito, sensación de cansancio y la falta de concentración ${ }^{16}$. Por lo tanto, las personas con discapacidad física deben atravesar por un periodo de aceptación y adaptación para tener una adecuada estabilidad emocional con el objetivo de fomentar su seguridad para la integración social y laboral.

Además en la investigación se demostró que $81 \%$ de la poblacion Siempre se muestran motivados a la hora de realizar algun tipo de actividad. La motivacion es aquella condicion interna descrita como anhelos, la voluntad de ejercer altos niveles de esfuerzo hacia las metas organizacionales, condicionadas por la habilidad del esfuerzo de satisfacer alguna necesidad individual 17 .

En el conjunto de las necesidades del ser humano, está enmarcada la de motivación personal como profesional18, ya que las necesidades se convierten en el motor que impulsa a salir adelante, es por ello que las personas con discapacidad física necesitan atravesar un periodo de adaptación en la cual busca la ayuda de sus seres más cercanos, para de ésta manera poder enfrentar a la sociedad con una óptima estabilidad física y mental y así poder continuar con sus metas personales y profesionales.

Cuando estas personas logran superar el periodo de adaptacion, demuestran seguridad y aunado a la presencia de factores protectores como el apoyo familiar, social y amical, logran adaptar un mecanismo de afrontamiento que les permite desenvolverse laboralmente.

Así mismo para un desenvolvimiento laboral eficiente es importante la apariencia física siendo un factor predisponente para la aceptación total de la persona, analizando el Gráfico $\mathrm{N}^{\circ} 2$ Dimensión Bienestar Física se encontró que el 97\% de la población manifiestan que siempre sus problemas de salud le producen dolor y malestar, al realizar cualquier tipo de actividad. El dolor agudo es aquel que amenaza seriamente la recuperación de un paciente, al interferir en su 
capacidad para estar activo e implicarse en su autocuidado; la rehabilitación puede retrasarse y la hospitalización prolongarse si el dolor agudo no es controlado ${ }^{19}$ Es por ello que las personas suelen recurrir a establecimientos de salud para poder tratar y controlar los episodios de malestar que aquejan su integridad fisica.

Evidenciandosé asi que el 56\% de las personas con discapacidad física Algunas veces, presentan dificultad de acceso a recursos de atención sanitaria, viendose afectado el control periódico y oportuno para su bienestar multidimensional.

Es ahí a donde esto nos lleva a otro dato importante en la presente investigación, que solo el $79 \%$ de la población en estudio manifiestan que nunca y algunas veces su estado de salud le permite llevar una actividad normal, evidenciadosé una cifra alarmante.

Es por ello que las personas que presentan discapacidad, sus actividades cotidianas se tornan mas complejas, ya que los discapacitados tienden a ser más dependientes de medios fisicos para el desarrollo de sus actividades diarias, dicho esto se obtuvo que el $49 \%$ de las personas manifiestan que Frecuentemente disponen de ayudas técnicas, lo cual les ayuda a tener mayor independencia, satisfaciendo así sus necesidades fisiologicas y de autorealización, con el objetivo de poder integrarsé de una manera mas eficiente al campo laboral.

Es asi que en la presente investigación en el Gráfico $\mathrm{N}^{\circ} 3$ en la dimensión Bienestar Material se encontró que el $42 \%$ de las personas con discapacidad física frecuentemente disponen de los recursos económicos necesarios para cubrir sus necesidades básicas. Es decir la cantidad de dinero que dispone un individuo con la finalidad de que sea utilizado en un determinado tiempo sin aumentar o disminuir sus activos netos 20 .

Los recursos materiales influyen en el comportamiento social y en la calidad de vida de las personas con discapacidad física, ya que la economia es utilizada para cubrir las necesidades basicas, fisiológicas, social y personales, permitiendo de esta manera obtener un lugar adecuado de acuerdo a sus limitaciones, obteniendo una mayor facilidad en su desplazamiento para realizar sus activdades diaras en el hogar. Esta disposicion de recursos se refleja en donde solo el $39 \%$ de personas Siempre perciben que el lugar en donde viven está adaptado a sus necesidades.

En la actualidad las personas con discapacidad física no cuentan con la economia suficiente para adaptar sus viviendas de acuerdo a la limitacion que presentan, es por ello que una cantidad considerable del $50 \%$ de personas encuestadas (18\% y $32 \%$ ) manifiestan que Frecuentemente y Siempre el lugar donde viven les impiden llevar un estilo de vida saludable. ${ }^{21}$

Es por ello que para el bienestar óptimo de la personas con discapacidad física es fundamental que tanto en su hogar como en su centro laboral cumplan con ciertos requisitos (rampas, barandales, ascensor, etc) y de esta manera pueda cumplir eficazmente con sus labores. Por lo que en nuestro medio, el bienestar óptimo de las personas con discapacidad fisica, se ve vulnerado, dado que las instituciones no cuentan con estas normas de seguridad, tal como la evidencia esta investigacion, donde se obtuvo que solo el $8 \%$ de personas manifiestan que Siempre el lugar donde trabajan cumplen con las normas de seguridad.

Ante lo expuesto se concluye que las entidades tanto publicas como privadas no le dan la debida importancia a el entorno en donde se deselvuelven las personas con discapacidad física impidiendo de esta manera su desarrollo biopsico-social.

Respecto al análisis del Gráfico $\mathrm{N}^{\circ} 4$, de la Dimensión Relaciones Interpersonales en donde el 77\% de las personas con discapacidad fisica siempre mantienen la relacion que desea con su familia. Por otro lado en la actualidad se evidencia casos de discriminación a las personas con discapacidad fisicia por parte de los familiares, ya 
que no pueden afrontar con la limitacion que le antecede a su familiar, conllevando de esta manera al maltrato tanto fisico como psicologico de las personas, es por ello que en la investigacion se obtuvo que el $98 \%$ de las personas manifiestan que Siempre se sienten infravalorados por un familiar y el $83 \%$ nunca suelen sentirse queridos por las personas importantes para el, es por ello que se evidencia que la persona con discapacidad son mas vulnerable ante la sociedad, por no contar con el apoyo que la familia y el entorno social les debe de brindar.

No obstante si la persona con discapacidad logra una integración laboral, social y funcional, podrá interrelacionarse adecuadamente con su entorno, manteniendo una buena relacion interpersonal tanto familiar como con la sociedad, por ello se obtuvo que sólo el 36\% frecuentemente matienen una buena relacion con sus compañeros de trabajo . 22

En nuestro sistema de organización social, disponer de un empleo es un principio fundamental, tanto para la integración social del sujeto como para el desarrollo de la personalidad del discapacitado.

El empleo constituye, por tanto, una importante vía de desarrollo personal y una incorporación plena a nuestra sociedad. En este sentido, el trabajo más que un fin en sí mismo es un medio para conseguir diferentes aspectos como son: una imagen más positiva y útil de uno mismo, el reconocimiento social, la estructuración del tiempo, la pertenencia a un grupo social, el aumento en la cantidad y calidad de interacciones sociales y la independencia económica, es por ello que en el Gráfico $N^{\circ} 5$ Dimensión Desarrollo Personal, el 93\% Frecuentemente y Siempre en el trabajo a donde acuden, toman en consideracion su desarrollo personal y el aprendizaje de habilidades nuevas.

Sin embargo existe factores predisponentes que puede conllevar a una insatisfaccion laboral de las personas con discapacidad, ya sea por problemas fisicos, emocionales o remunerativos, es por ello que obtenemos que una cifra considerable de personas (47\%) manifiestan que Nunca y Algunas veces se muestran desmotivados con su trabajo, conllevando de esta manera a realizar un trabajo incompetente, siendo percibidos por ellos mismo, donde el 61 \%de las personas discapacitadas percibe que Nunca desarrollan su trabajo de manera competente y responsable. 16

Es por ello que las instituciones como parte de su compromiso deben seguir ayudando a las personas con discapacidad fisica, a un adecuada adaptacion, brindandoles la confianza sufuciente para que estos puedan desarrollar sus habilidades y destrezas en el campo laboral, sin tener miedo a equivocarse o limitarse. Esta situación es reflejada por un $62 \%$ de la poblacion que Nunca muestran dificultad para adptarse a las situaciones que se les presentan.

Muchas veces la dificultad para la adecuada adaptacion se ve ligada al rechazo y la discriminacion que existe a nivel social por un desconocimiento o por una errónea idea de lo que es un persona con discapacidad fisica, por ello en el Gráfico N 6 Dimensión inclusión Social, el 49\% de la poblacion frecuentemente manifiesta que existen barreras fisicas, culturales o sociales que dificultan su integracion social.

Es por esto que existe leyes que amparan al discapacitado, como lo es la Ley General de la persona con discapacidad $\mathrm{N}^{\circ} 29973$, ley que nos habla sobre el reconociemiento a la persona con discapacidad en todos los organismos del estado como son, inclusióon social, inclusión en el area laboral, tratamientos y prevención para los casos de temprana edad, represetacion eletoral, facilidades para acudir a los eventos culturales, educativos y recreativos, capacidad juridica, asistencia y programas de salud, alimentacion, vestido y vivienda ${ }^{23}$. Asimismo es $48 \%$ de las 
personas con discapacidad fisicas mencionan que siempre utilizan entornos comunitarios.

Sin embargo es en estos entornos comunitarias que el $55 \%$ frecuentemente suelen sentirse rechazados o discriminados por los demás, tal es así que según la Organización de las Naciones Unidas la convención sobre los derechos de las personas con discapacidad es la respuesta de la comunidad internacional a la larga historia de discriminacion, exclusión y deshumanización de las personas con discapacidad 24. Es ante tal rechazo que incluye entre sus principios generales: la no discrimincion, la participacion e inclusion plenas y efectivas en la sociedad.

Pero actualmente se evidencian los altos índices de discriminación y el poco interés de los empleadores en fomentar el desarrollo biopsicosocial de las personas con discapacidad física en su centro laboral. Por tal motivo se encontró que el $86 \%$ alegan que al trabajo a donde acuden Nunca fomentan su participación en diferentes actividades en la comunidad.

Es por ello que ante esta situación la ley que ampara al discapacitado ha servido para que un cierto porcentaje de las instituciones tanto pública como privadas, tomen conciencia en brindar las medidas adecuadas ya sea en infraestructura, o un adecuado clima organizacional y de esta manera llegar a fomentar la inclusión y el desarrollo de la persona discapacitada, por ello en el Gráfico $\mathrm{N}^{\circ} 7$ Dimensión Autodeterminación se encontró que el $39 \%$ de las personas con discapacidad física, Siempre al trabajo al donde acuden tienen en cuenta sus preferencias, y el $32 \%$ manifiestan que Siempre tiene metas, objetivos $\mathrm{y} / \mathrm{o}$ intereses personales, en donde las instituciones son parte de este desarrollo personal y laboral.

Pero éste desarrollo debe involucrar también a la familia, quienes son la base fundamental para el desarrollo personal y el crecimiento profesional, sin embargo en la actualidad algunas personas con discapacidad física carecen de este apoyo evidenciándose un inestabilidad emocional, vulnerando de esta manera su autonomía y su independencia es por ello que se encontró que el $36 \%$ de la población en estudio mencionan que Algunas veces otras personas deciden como gastar su dinero, y el $63 \%$ manifiestan que Algunas veces eligen con quien vivir. Ante esta situación el profesional de la salud debe de ser hincapié en la importancia del rol familiar en la vida de una persona con discapacidad física para el bien de la misma y de esta manera poder enfrentar a la sociedad con un carácter muy bien posicionado.

Por otro lado las instituciones ya sean públicas o privada deben hacer valer su derechos de las personas con discapacidad física, ya que así está estipulado en la Ley general del discapacitado, es así que también es un deber de estas personas saber cuáles son sus derechos y sus deberes para hacer valer los mismos, analizando el Gráfico $\mathrm{N}^{\circ} 8$ Dimensión Derechos, se obtuvo que el $48 \%$ si disponen de información sobre sus derechos fundamentales como ciudadanos, a la vez el $37 \%$ de las personas mencionan que al trabajo a donde acuden Siempre defienden y respetan sus derechos, aunque es un porcentaje un poco bajo podemos evidenciar que actualmente si existe instituciones que protegen la calidad laboral de sus trabajadores discapacitados, obteniéndose que el $83 \%$ de las personas con discapacidad física mencionan que Nunca sufren situaciones de explotación, violencia o abuso.

Pero los derechos no solo se hacen valer en una institución laboral, sino que también es deber de la sociedad integrar favorablemente a estas personas para que disfruten de un entorno saludable y así puedan desarrollarse eficazmente, es así que el 54\% Algunas veces muestran dificultades para defender sus derechos 25 . 


\section{Conclusiones}

$\checkmark$ De acuerdo al Índice de Calidad de Vida, se obtuvo que las dimensiones Autodeterminación, R. Interpersonales, D. Personal e Inclusión social, se encuentran antes de la media estándar de la calidad de vida esperada (10 DE + - 3); sin embargo, las dimensiones B. Emocional, B. Físico, B. Material y Derechos se encuentran por debajo de la media estándar de la calidad de vida esperada

$\checkmark$ Las dimensiones Autodeterminación, R. Interpersonales, D. Personal e Inclusión social, se encuentran antes de la media estándar esperada; sin embargo, las dimensiones $B$. Emocional, B. Físico, B. Material y Derechos se encuentran por debajo de la media estándar esperada (10 DE + - 3), es decir son las más afectadas en su calidad de vida, encontrándose dificultades en el desarrollo bio - psicológico de las personas con discapacidad física.

1. Con respecto a la dimensión Bienestar Emocional las personas con discapacidad física siempre el $81 \%$ se encuentra motivado al realizar algún tipo de actividad, sin embargo, algunas veces el $58 \%$ presentan síntomas de depresión, el 59\% frecuentemente y algunas veces muestran sentimientos de incapacidad e inseguridad y $45 \%$ algunas veces y nunca se encuentran satisfechos con su vida presente, de esta manera se ve afectado y vulnerado el bienestar emocional conllevando así a un incumplimiento en sus quehaceres laborales.

2. En la dimensión Bienestar Físico las personas con discapacidad siempre el $91 \%$ sus hábitos de alimentación son saludables, sin embargo, el $79 \%$ nunca y algunas veces les permite llevar una actividad normal. El 97\% siempre y frecuentemente sus problemas de salud le producen dolor y malestar y el $56 \%$ algunas veces tienen dificultad de acceso a la atención sanitaria, conllevando a que su estado de salud empeore si no se le brinda una adecuada calidad de vida en su centro laboral
A continuación, se presentan las conclusiones derivadas de esta investigación:

3. En la dimensión Bienestar material las personas con discapacidad física siempre (81\%) disponen de recursos necesarios para cubrir sus necesidades básicas logrando de esta manera una independencia economía. Sin embargo para un porcentaje de las personas con discapacidad algunas veces (50\%) el lugar en donde viven les impide llevar un estilo de vida saludable, y el $73 \%$ manifiesta que siempre el lugar en donde trabajan cumplen con las normas de seguridad, resguardando así la seguridad, la salud integral y la correcta inclusión en el centro laboral siendo ello parte de la dimensión de Derechos, en los cuales el $48 \%$ disponen de información sobre sus derechos fundamentales como ciudadano y solo el $54 \%$ muestran dificultades para defenderlos por lo que estas personas deben empoderarse de conocimiento sobre los derechos y leyes que amparan al discapacitado, y de esta manera evitar abusos laborales y sociales

4. En la dimensión Relaciones interpersonales las personas con discapacidad física siempre (98\%) manifiesta sentirse infravalorado por algún familiar, así mismo el $83 \%$ nunca manifiesta sentirse querido para las personas importantes para él y el $82 \%$ nunca y algunas veces realiza actividades que le gusta con otras personas, conllevando así a una desconfianza en sí mismo, miedo ante las relaciones personales y exclusión social, afectando a la vez la dimensión de Inclusión social donde se evidencia que el $92 \%$ de la población encuentran barreras físicas, culturales y sociales que dificultan su inclusión social, así mismo el $88 \%$ frecuentemente suelen sentirse rechazados o discriminados por los demás, reflejándose la poca concientización de la población y de los centros laborales.

5. En la dimensión Desarrollo personal las personas con discapacidad física 
frecuentemente (87\%) al trabajo a donde acuden toman en consideración su desarrollo personal y aprendizaje de habilidades nuevas desarrollando en ellos confianza suficiente para desenvolverse en el entorno social. Sin embargo, el $53 \%$ se

6. En la dimensión Autodeterminación las personas con discapacidad física siempre (53\%) el centro laboral a donde acuden tiene en cuenta sus preferencias, el $91 \%$ nunca organiza su propia vida y el $80 \%$ siempre otras personas deciden su vida profesional. Por otro lado, mencionan que algunas veces (62\%) otras personas deciden como gastar su dinero, siendo manipulados debido a su condición física, de esta manera la persona con discapacidad física se ve vulnerada en la toma de sus propias decisiones.

\section{Bibliografía}

1. Kozier B. Fundamentos de Enfermería. Vol. 1 y 2. Editorial MC Graw Hill. México, 1999.

2. Ley General de la Persona con Discapacidad, Ley $\mathrm{N}^{\circ}$ 27050, 18-12-1998, Boletín Oficial Diario "El Peruano $n^{\circ} 6770$ (06.01.99) URL disponible en https://www.mef.gob.pe/contenidos/servicios _web/conectamef/pdf/normas_legales_2012/ NL20121224.PDF.

3. Guerrero A. "Mejorando la calidad de vida de personas seropositivas a través del modelo de calidad de vida relacionado con salud, Chiclayo- 2013 [Tesis para Licenciatura]. Escuela de Enfermería -Universidad Católica Santo Toribio de Mogrovejo. 2014

4. Instituto Nacional de Estadística e Informática [internet], Lima-Perú, [actualizado 02-122013, citado 19-05-2015] En el Perú 1 millón 575 mil personas presentan algún tipo de discapacidad [aprox, 4 pantallas] Disponible en:http://www.inei.gob.pe/media/MenuRecur sivo/noticias/np-178-2013.pdf

5. Herrero M. Calidad de Vida de las Personas con Discapacidad en la Provincia de Huesca. Cadis.2005. 13-20. muestra desmotivado con su trabajo y perciben que nunca (86\%) desarrollan su trabajo de manera responsable $y$ competente, esto va relacionado a lo poca motivación e integración de estas personas en su centro laboral.

6. Cardona D, Agudelo A, Restrepo L, Sánchez L, Segura A. Calidad de Vida de las Personas en Situación de Discapacidad Física. Medellín, 2011. CES Salud Publica; 2014, 5, 137-146.

7. Gómez L, Verdugo $M$, Arias B, Irurtia J. behavioral psicología/psicología conductual: Evaluación de los derechos de las personas con Discapacidad intelectual; Universidad de Valladolid; INICO. Universidad de Salamanca (España). 2011; 19(01): 207-222

8. Herrero M, Elías V. Calidad de vida de las personas con discapacidad física en la provincia de Huesca. Zaragoza (España).2008. ISBN: 978-84-692-3237-8

9. Hernández R, Fernández C, Baptista $P$. Metodología de la investigación. $5^{\circ}$ ed. México. 2003.

10. Consejo Nacional para la integración de la persona con discapacidad (CONADIS). [Fecha de acceso 26 de Marzo del 2015]. URL disponible en: http://www.conadisperu.gob.pe/

11. Guerrero A. "Mejorando la calidad de vida de personas seropositivas a través del modelo de calidad de vida relacionado con salud, Chiclayo- 2013 [Tesis para Licenciatura]. Escuela de Enfermería -Universidad Católica Santo Toribio de Mogrovejo. 2014

12. Aton Almenara P. Enfermería: Ética y Legislación en Enfermería. Serie Manuales de Enfermería, España, 2003.

13. Verdugo A, Arias M, Schalock R. Escala Gencat: Manual de aplicación de la Escala Gencat de la calidad de vida. Acción Social I Ciuta Dania.2009. Vol. 1 (1): 69-76.

14. Urzua M, Caqueo A. Calidad de vida: Una revisión teórica del concepto. Ter Psicol [online]. 2012, vol.30, n.1 [citado 2017-0328], pp.61-71. Disponible en: $<$ http://www.scielo.cl/scielo.php?script=sci_a 
rttext\&pid=S0718-

$48082012000100006 \&$ lng $=e s \& n r m=$ iso $>$.

ISSN

0718-4808.

http://dx.doi.org/10.4067/S071848082012000100006.

15. Latorre A. Bienestar emocional y expresión conductual en las personas con discapacidad intelectual. [online]. Julio- Diciembre 2010. Pp.51-60.ISSN: 1888-4857. Disponible en : file:///C:/Users/user/Downloads /DialnetBienestarEmocionalYExpresionConductualEnLa sPersona-3276331.pdf

16. Organización Mundial de la Salud. Definición de Depresión. [Sede web]*. [Actualizada en el 2015: acceso 15 de enero del 2016]. Disponible en: http://www.who.int/topics/depression/es/

17. Martínez M, Pérez A, Sanabria G. Interrelación dialéctica entre calidad de vida y motivaciones relativas a la salud. Rev Cubana Med Gen Integr [Internet]. 2010 Mar [citado 2016 Feb 01]; 26(1): Disponible en: http://scielo.sld.cu/scielo.php?script =sci_artt ext\&pid=S0864-

$21252010000100016 \&$ Ing =es.

18. Quintero J, Maslow A. y su teoría de la motivación humana. Seminario teorías y paradigmas educativos [en línea]. 2012. [ Fecha de acceso 10 de Mayo de 2016]; URL disponible

en: http//dosctorado.josequintero.net/

19. Ruzafa J, Damián J. Valoración de la discapacidad física: el índice de Barthel. Rev. Esp. Salud Pública [revista en la Internet]. 1997 Mar [citado 2016 Feb 09]; 71(2): 127-137. Disponible en: http://scielo.isciii.es / scielo.php?script=sci_art text\&pid $=$ S1 135 $57271997000200004 \& \operatorname{lng}=\mathrm{es}$

20. Rodríguez R. Vicente, Rojo P. Fermina, Fernández M. Gloria. Recurso económico y calidad de vida en la población mayor. Vol.: 69. agosto 2011 . ISSN:0034-9712

21. Orozco Juan Guillermo- OPS Perú. Hacia una vivienda saludable.

Organización
Panamericana de la Salud. Mayo 2012. ISBN: 978-9972-222-18-4.

22. Organización Mundial de la Salud. Definición Relaciones interpersonales. [ Sede Web]". [Actualizada en el 2014: acceso 12 de enero del 2016]. Disponible en: http://www.who.int/whr/2003/overview/es/i ndex2.html

23. Ley General de la Persona con Discapacidad, Ley $\mathrm{N}^{\circ} 27050,18-12-1998$, Boletín Oficial Diario "El Peruano $n^{\circ} 6770$ (06.01.99) URL disponible en https://www.mef.gob.pe/contenidos/servicios _web/conectamef/pdf/normas_legales_2012/ NL20121224.PDF

24. Organización de las naciones unidas. Convención sobre los derechos de las personas con discapacidad. [Sede Web]*. [Actualizada en el 2015: acceso 3 de Marzo del 2016]. Disponible en: http://www.un.org/esa/socdev/enable/docu ments/tccconvs.pdf

25. Lamas H. La situación de los discapacitados en el Perú: Exclusión/ inclusión de las personas con discapacidad, Rev. Cultura Perú. Vol.; 18, №18. Pág.: 242-259. [Internet] Año 2014. [ citado 2016 Mar 25]. Disponible en: www.revistacultura.com.pe/imagenes/pdf/ 18 $-13 . p d f$ 